\title{
Hemoperitoneum in Third Trimester of Pregnancy: Unusual Cause-Spontaneous Rupture of Uterine Varices
}

\author{
${ }^{1}$ Snehal Dhobale, ${ }^{2}$ Revathi S Rajan, ${ }^{3}$ Kamini A Rao
}

\begin{abstract}
Spontaneous uterine varices rupture is a very rare condition $(1 / 10,000$ pregnancies), leading to significant maternal and fetal morbidity and mortality. Though the exact etiology is still unknown, it seems to be associated with an increased in venous pressure. The rupture especially occurs in third trimester. We encounter a case of 31 years old primigravida with pain in abdomen since 1 day not relieved by medication. Abdominal ultrasound revealed hemoperitoneum. Emergency exploratory laparotomy revealed 1.5 litres of hemoperitoneum and confirmed the diagnosis of 'spontaneous rupture of uterine varices'. The clinical presentation of spontaneous rupture of uterine varices is not specific and clinical examination and ultrasonographic scanning may be insufficient for diagnosis. This rare condition has to be taken into account to the differential diagnosis in a pregnant woman with severe abdominal pain.
\end{abstract}

Keywords: Uterine varices, Hemoperitoneum, Abdominal ultrasound.

How to cite this article: Dhobale S, Rajan RS, Rao KA. Hemoperitoneum in Third Trimester of Pregnancy: Unusual Cause-Spontaneous Rupture of Uterine Varices. Int J Infertil Fetal Med 2014;5(2):66-68.

Source of support: Nil

Conflict of interest: None

Date of Received: $12-06-14$

Date of Acceptance: 05-08-14

Date of Publication: May 2014

\section{INTRODUCTION}

Spontaneous rupture of uterine varices in pregnancy is a rare condition (1/10,000 pregnancies $)^{1}$ leading to significant maternal and fetal morbidity and mortality. The exact etiology is still unknown, but it seems to be associated with an increase in venous pressure. The rupture is common in the third trimester. ${ }^{2,3}$ Establishing clinical diagnosis of this condition is difficult, but essential. The presenting symptoms are commonly nonspecific and the diagnosis is usually made

\footnotetext{
${ }^{1}$ Fellow, ${ }^{2}$ Senior Consultant, ${ }^{3}$ Medical Director

${ }^{1,3}$ Department of Reproductive Medicine, Milann-The Fertility Center, Bengaluru, Karnataka, India

${ }^{2}$ Department of Fetomaternal Medicine, Milann-The Fertility Center, Bengaluru, Karnataka, India
}

Corresponding Author: Snehal Dhobale, Fellow, Department of Reproductive Medicine, Milann-The Fertility Center Karnataka, Bengaluru, India, Phone: 08022011333, e-mail: snehswar08@gmail.com at laparotomy. ${ }^{4}$ The uniqueness of its presentation is in the fact that since 1950 , only eight cases have been reported. ${ }^{5}$ It mimics placental abruption having similar clinical presentation like acute abdominal pain, peritonitis, shock and fetal distress or death. ${ }^{6}$ The main objective of this case report is to discuss the diagnosis and management of a rare acute surgical condition in pregnancy which warrants immediate intervention.

\section{CASE OUTLINE}

A 31-year-old primigravida, 29+1 weeks natural conception, reported to outpatient department (OPD) with complaints of pain in upper abdomen since 1 day. Pre-existing risk factors included history of gastritis, subclinical hypothyroidism and diet controlled gestational diabetes mellitus. There was past history of laparotomy for left ovarian cystectomy and laparoscopic ovarian drilling for polycystic ovaries.

At presentation her vitals were stable. On per abdominal examination, epigastric tenderness was present. Uterus was corresponding to 30 weeks and was relaxed. Fetus was in breech presentation with fetal heart rate of $140 \mathrm{bpm}$. Cardiotocography was reactive and appropriate for the gestational age. Complete hemogram, serum electrolytes, liver and renal function tests on admission were normal except for a hemoglobin of $10.1 \mathrm{gm} \%$. The symptoms progressed gradually overnight and the pregnant mother continued to have epigastric pain as well as right hypochondriac and shoulder pain associated with positional discomfort on lying down. This triggered the possibility of acute cholecystitis/pancreatitis. Hence, she was shifted to tertiary care unit anticipating surgical or medical intensive care unit management after counselling implications.

Abdominal ultrasound revealed moderate free fluid in the peritoneal cavity, liver and gall bladder were normal and subsequently ultrasound guided tapping of fluid was performed which was hemorrhagic. Repeat hemogram after about 12 to 16 hour showed $\mathrm{Hb}: 4.5 \mathrm{gm} \%$ Platelet count: $44,000 / \mathrm{cmm}$. International normalised ratio (INR) was deranged. Dengue nonstructural protein 1 (antigentesting for dengue) (NS1) antigen test was negative. She was afebrile and normotensive. Patient was supported with IV fluids, IV antibiotics and prophylactic steroid were administered for fetal lung maturity. A decision for exploratory laparotomy with 
lowere segment cesarean section (LSCS) with simultaneous correction of hematological parameters with blood products was taken.

The relevent intraoperative findings were 1.5 liters of hemoperitoneum. There was evidence of ruputre of dilated tortous vessels (uterine varices) over anterolateral aspect of uterus (Fig. 1). Rest of the abdomen including adnexa was normal. There was no other obvious source of bleeding. A male baby weighing $1.3 \mathrm{~kg}$ was delivered and shifted to neonatal intensive care unit (NICU). Hemostasis was achieved with stay sutures over dilated varices (Fig. 2).

The projected intraoperative blood loss was 2.5 liters. She was stabilized with intra and postoperative transfusions which included 4 units whole blood, 1 unit packed cell volume, 6 units platelet, 4 units fresh frozen plasma. Postoperatively patient was shifted to ICU for 3 days followed by in ward for 2 weeks. Baby required ventilation for 24 hours and then NICU stay for subsequent 2 weeks. She was discharged with baby after 15 days. The postoperative recovery as well as subsequent follow-ups were uneventful.

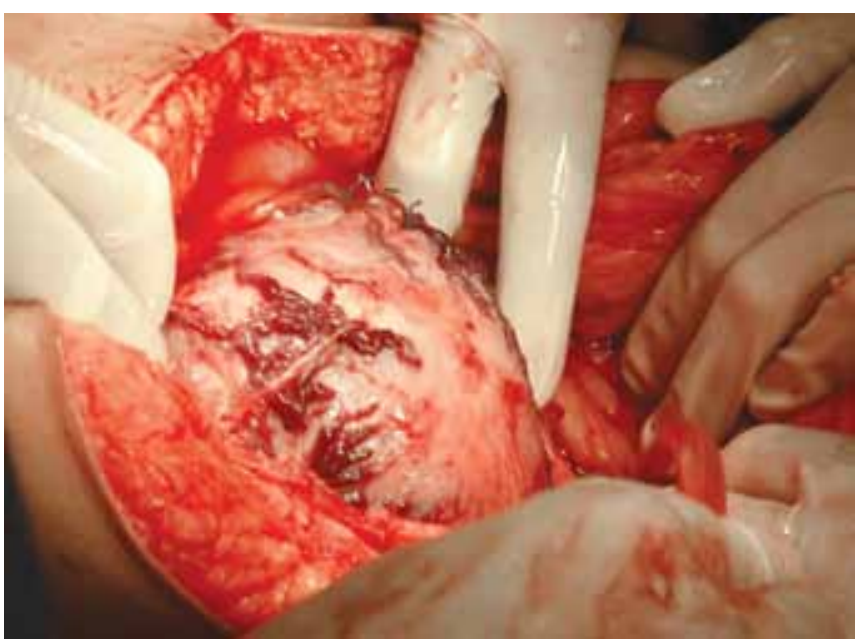

Fig. 1: Dilated tortous vessels (uterine varices) over anterolateral aspect of uterus

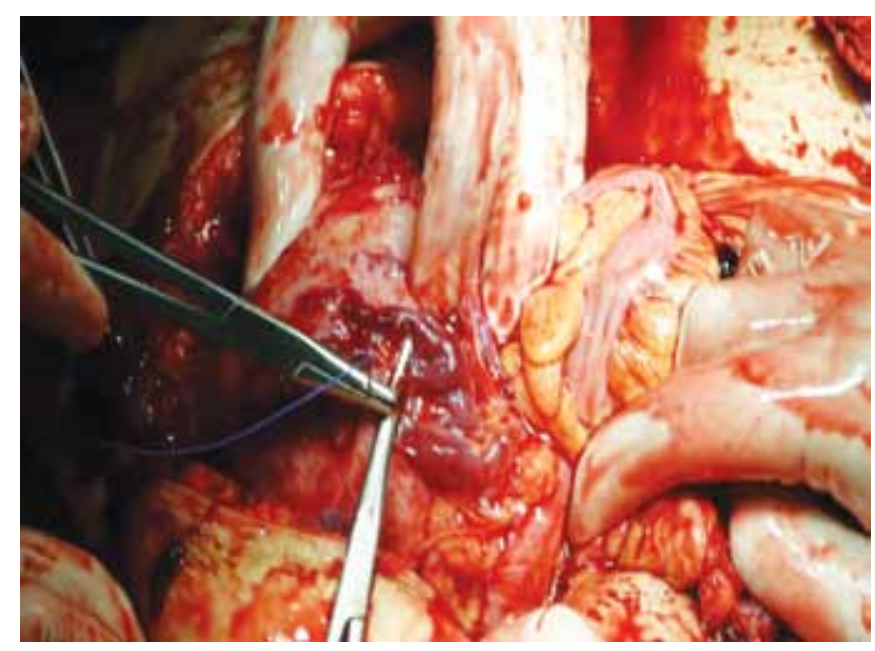

Fig. 2: Hemostasis with stay sutures

\section{DISCUSSION}

Spontaneous uterine venous rupture in pregnancy is a relatively rare $(1 / 10,000$ pregnancies) but life threatening condition. The etiology is, as yet, poorly understood and subject to speculation. The rupture especially occurs in third trimester. The most frequent site of the rupture is in the broad ligament. Owing to recent advances which improved anesthetic, resuscitative, and operative techniques, maternal mortality has been considerably lowered from 49 to $3.6 \%{ }^{4}$ Perinatal mortality, however, remains high at $31 \%$. Of cases reported in the literature, $61 \%$ occurred before labor, $19 \%$ were intrapartum, and $21 \%$ were puerperal. ${ }^{3}$ In symptomatic cases, rupture of an utero-ovarian vessel leads to intraperitoneal bleeding or retroperitoneal hematoma, or both.

Diagnostic dilemma: The presenting symptoms are commonly nonspecific. Clinical presentations are usually signs of hypovolemic shock with severe abdominal pain and reduction in hemoglobin levels without obvious bleeding. Establishing clinical diagnosis of this condition is difficult, but significant.

Differential diagnosis: The diagnosis of this condition is rarely made before exploratory laparotomy and is most frequently misdiagnosed as abruptio placentae, ${ }^{6}$ especially in the absence of ultrasonographic evaluation. Other diffe-rential diagnoses include uterine rupture, HELLP syndrome, abdominal pregnancy, rupture of the spleen or liver and ruptured appendix.

Hodgkinson and Christiansen suggested the possible cause was dilated utero-ovarian vessels resulting from the increased physiological demands of pregnancy and muscular activities, such as coughing, defecation, coitus, or pushing during the second-stage of labor, which cause a sudden rise in venous pressure. ${ }^{7}$ The tortuous nature, lack of valves, and repeated distension of these vessels during pregnancy are thought to make them predisposed to rupture. However, these tortuous, dilated vessels are quite commonly observed in many normal pregnancies during cesarean section. Thus, the possible existence of additional vascular defects in the affected pregnancies could be suspected. Recent case reports support this idea and suggest a possible origin from decidualized endometriosis on the utero-ovarian vessel wall. ${ }^{4}$

Emergency laparotomy is warranted in all such clinical situations possibly due to extensive internal bleeding so as to establish the correct diagnosis. Preoperative ultrasonographic evaluation often adds value and aids the diagnosis of hemoperitoneum. The situation could worsen after the onset of labor as the pain experienced due to uterine contractions may mask the peritoneal irritation from internal bleeding. The expulsive effort during second stage of labor could 
increase the risk of venous rupture. Hence, abdominal ultrasound in pregnant women with acute abdominal symptoms is essential to reach a conclusive diagnosis.

Preoperative diagnosis could be made only with a strong clinical suspicion supported with appropriate investigation and ultrasound findings. Prompt recognition and early diagnosis followed by hemodynamic stabilization and laparotomy with appropriate patient counseling is considered vital in the management of acute surgical emergencies complicating pregnancy.

\section{REFERENCES}

1. Hashimoto K, Tabata C, Ueno Y, Fukuda H, Shimoya K, Murata Y. Spontaneous rupture of uterine surface varicose veins in pregnancy: a case report. J Reprod Med 2006 Sep;51(9):722-724.

2. Nakaya $Y$, Itoh H, Muramatsu K, Otome M, Kobayashi Y, Hirai K, Uchida T, Suzuki K, Sugihara K, Kanayama N. A case of spon- taneous rupture of a uterine superficial varicose vein in midgestation. J Obstet Gynaecol Res 2011 Aug;37(8):1149-1153.

3. González Rosales R, Cerón Saldaña MA, Ayala Leal I, Cerda López JA. Uterine vessels spontaneous rupture during pregnancy: case report and literature review. Ginecol Obstet Mex 2008 Apr;76(4):221-223.

4. Andrés-Orós MP, Server-de Castro L, Roy-Ramos V, VelaLete A. Spontaneous uterine venous rupture during pregnancy. Description of two cases and bibliographical review. Ginecol Obstet Mex 2010 Feb;78(2):128-131.

5. Pittion S, Refahi N, Barjot P, Von Theobald P, Dreyfus M. Spontaneous rupture of uterine varices in the third trimester of pregnancy. J Gynecol Obstet Biol Reprod (Paris) 2000 Dec; 29(8):801-802.

6. Subedi S, Banerjee B. Spontaneous hemoperitoneum masquerading placental abruption: a case report. NJOG 2012 Jul-Dec; 7(2):50-51.

7. Marrow AE. Rupture of varicose veins of the uterus associated with pregnancy. BJOG: An International Journal of Obstetrics and Gynaecology 1960;67(5):792-795. 